\title{
Synaptic plasticity in vivo: more than just spike-timing?
}

\author{
Jan M. Schulz* \\ Department of Physiology, University of Bern, Bern, Switzerland \\ *Correspondence: schulz@pyl.unibe.ch
}

\section{A commentary on}

Questions about STDP as a general model of synaptic plasticity.

by Lisman, J., and Spruston, N. (2010). Front. Syn. Neurosci. 2:140. doi: 10.3389/ fnsyn.2010.00140.

In their recent Perspective Article, Lisman and Spruston (2010) succinctly describe the crucial shortcomings of spike-timingdependent plasticity (STDP) to serve as a unifying principle of synaptic plasticity. In particular, it is convincingly argued that postsynaptic depolarization rather than a somatic action potential (AP) is necessary and sufficient for the explanation of most results that have usually been interpreted within the STDP framework. I would like to add that we know even less about the importance of single backpropagating APs for synaptic plasticity in vivo.

Direct evidence for STDP in vivo is limited and suffers from the fact that the used protocols significantly deviate, more often than not, from the traditional pairing of single pre- and postsynaptic spikes (Shulz and Jacob, 2010). Thus, many studies use long-lasting large-amplitude postsynaptic potentials (PSP), and pairing usually involves multiple postsynaptic spikes or high repetition rates. Our own experience from cortico-striatal synaptic plasticity experiments indicates that classic STDP may be less effective in vivo than commonly expected (Schulz et al., 2010). A limited number of pairings (60 times) of cortical PSPs with a single current-induced postsynaptic AP at a slow rate (every $5 \mathrm{~s}$ ) resulted in smaller and much more variable synaptic plastic changes than in previous in vitro studies that used comparable protocols (Pawlak and Kerr, 2008; Fino et al., 2010; Schulz et al., 2010). On the other hand, we did find that regular somatic AP discharge during the pre-post pairings was necessary for any synaptic potentiation and that the direction of induced plasticity was crucially dependent on the relative timing of the synaptic inputs to the somatic AP on a millisecond-timescale (Schulz et al.,
2010). This is strikingly different from previous in vitro studies that suggested that even large current-induced subthreshold depolarizations are sufficient to induce synaptic changes at the cortico-striatal synapse over a wider range of timing intervals (Fino et al., 2009). These results demonstrate that one has to be very careful with extrapolating from in vitro results to the in vivo situation. Several factors have to be taken into account.

First, most in vitro studies on STDP use cell cultures or acute slices from young animals, where neural circuits are naturally more plastic than in the adult brain (e.g., Meredith et al., 2003). While results obtained from such preparation are important for our understanding of developmental processes, their relevance to our concepts of learning is less clear.

Second, the network state in vitro is fundamentally different from the in vivo situation. In acute slices in particular, background synaptic activity is almost absent. Pairing of unitary PSPs with single postsynaptic APs is usually insufficient to induce STDP under these conditions (Markram et al., 1997; Kampa et al., 2006). Only if large PSPs and/or bursts of postsynaptic APs are evoked, that induce a dendritic calcium spike, STDP will be observed. These observations directly support Lisman and Spruston when they argue that active dendritic mechanisms of depolarization like NMDA and $\mathrm{Ca}^{2+}$ spikes may be more relevant for synaptic plasticity than backpropagating APs. In vivo, however, most telencephalic neurons are in a high-conductance state (Rudolph et al., 2005). In this state, the input resistance is dramatically decreased in soma and dendrites. At the same time, active dendritic mechanisms may become more readily available due to the depolarized membrane potential. Therefore, it is not trivial to predict how this state will affect STDP. In the only study so far, that simulated the high-conductance state with the dynamic clamp technique in vitro (Delgado and Desai, 2008), classic STDP became a lot less effective and the timing window was greatly reduced.
Third, natural inhibition is often pharmacologically blocked in in vitro studies on STDP. Yet, inhibition powerfully regulates STDP: in the hippocampus, STDpotentiation cannot be induced using single postsynaptic spikes during the pairing in slices from young adult mice; however, STD-potentiation can be re-established by either using postsynaptic spike bursts or by blocking GABA-mediated inhibition (Meredith et al., 2003). In the striatum, blocking GABA-mediated inhibition results in the reversal of the STDP-timing rule in slices from juvenile rats (Fino et al., 2010). We think that the reversed timing rule of the narrower STDP-window that we observed in the adult striatum in vivo may also be a result of lateral inhibitory mechanisms (Schulz et al., 2010).

A fourth factor is neuromodulation. Neuromodulators like dopamine could regulate when strong postsynaptic depolarization is capable of changing the weight of active synapses and when not (Pawlak and Kerr, 2008; Schulz et al., 2010). This could be a result of the modulation of intrinsic properties and synaptic transmission; at the same time, neuromodulators can also directly interact with the biochemical pathways that mediate synaptic plastic changes (Valjent et al., 2005). Neuromodulatory regulation could be an elegant solution to prevent regular readout of stored information, in the form of somatic spiking, from altering the stored information. In contrast, Lisman and Spruston's suggestion that dendritic spikes could regulate synaptic plasticity without being affected by regular read-out seems improbable, since dendritic spikes are also very likely to be an essential part of the read-out process of information stored in distal synapses (Rudolph et al., 2005; Larkum et al., 2009).

In summary, it seems probable that synaptic plasticity in the intact brain is governed by rules that are much more complex than the traditional interpretation of STDP. In my opinion, it should be of concern to all those who model animal learning that, 
in general, studies using less physiological conditions appear to be more successful at reproducing classic STDP. Instead, the defining postsynaptic event may vary between simple postsynaptic depolarization, local dendritic spike, single backpropagating APs and AP bursts, depending on neuron type, developmental stage and network state. As pointed out by Lisman and Spruston, the precise timing between pre- and postsynaptic events will remain of crucial importance. However, it becomes increasingly evident that the outcome will not only depend on these but also of converging inhibitory and neuromodulatory inputs.

\section{REFERENCES}

Delgado, J. Y., and Desai, N. S. (2008). In vivo-like conductances limit spike-timing dependent plasticity. Soc. Neurosci. Abstr. 39, 40-43.

Fino, E., Deniau, J.-M., and Venance, L. (2009). Brief subthreshold events can act as Hebbian signals for long-term plasticity. PLoS ONE4, e6557.doi: 10.1371/ journal.pone.0006557.
Fino, E., Paille, V., Cui, Y. H., Morera-Herreras, T., Deniau, J. M., and Venance, L. (2010). Distinct coincidence detectors govern the corticostriatal spike timing-dependent plasticity. J. Physiol. (Lond.) 588, 3045-3062.

Kampa, B. M., Letzkus, J. J., and Stuart, G. J. (2006). Requirement of dendritic calcium spikes for induction of spike-timing-dependent synaptic plasticity. $J$. Physiol.-Lond. 574, 283-290.

Larkum, M. E., Nevian, T., Sandler, M., Polsky, A., and Schiller, J. (2009). Synaptic integration in tuft dendrites of layer 5 pyramidal neurons: a new unifying principle. Science 325, 756-760.

Lisman, J., and Spruston, N. (2010). Questions about STDP as a general model of synaptic plasticity. Front. Syn. Neurosci. 2:5. doi: 10.3389/fnsyn.2010.00140.

Markram, H., Lubke, J., Frotscher, M., and Sakmann, B. (1997). Regulation of synaptic efficacy by coincidence of postsynaptic APs and EPSPs. Science 275, 213-215.

Meredith, R. M., Floyer-Lea,A. M., and Paulsen, O. (2003) Maturation of long-term potentiation induction rules in rodent hippocampus: role of GABAergic inhibition. J. Neurosci. 23, 11142-11146.

Pawlak, V., and Kerr, J. N. D. (2008). Dopamine receptor activation is required for corticostriatal spike-timingdependent plasticity. J. Neurosci. 28, 2435-2446.

Rudolph, M., Pelletier, J. G., Pare, D., and Destexhe, A. (2005). Characterization of synaptic conductances and integrative properties during electrically induced EEG-activated states in neocortical neurons in vivo. J. Neurophysiol. 94, 2805-2821.

Schulz, J. M., Redgrave, P., and Reynolds, J. N. J. (2010). Cortico-striatal spike-timing dependent plasticity after activation of subcortical pathways. Front. Syn. Neurosci. 2:23. doi: 10.3389/fnsyn.2010.00023.

Shulz, D. E., and Jacob, V. (2010). Spike timing dependent plasticity in the intact brain: counteracting spurious spike coincidences. Front. Syn. Neurosci. 2:137. doi: 10.3389/fnsyn.2010.00137.

Valjent, E., Pascoli, V., Svenningsson, P., Paul, S., Enslen, H., Corvol, J. C., Stipanovich, A., Caboche, J., Lombroso, P. J., Nairn, A. C., Greengard, P., Herve, D., and Girault, J. A. (2005). Regulation of a protein phosphatase cascade allows convergent dopamine and glutamate signals to activate ERK in the striatum. Proc. Natl. Acad. Sci. U.S.A. 102, 491-496.

Received: 08 October 2010; accepted: 09 November 2010; published online: 22 November 2010.

Citation: Schulz JM (2010) Synaptic plasticity in vivo: more than just spike-timing? Front. Syn. Neurosci. 2:150. doi: 10.3389/fnsyn.2010.00150

Copyright () 2010 Schulz. This is an open-access article subject to an exclusive license agreement between the authors and the Frontiers Research Foundation, which permits unrestricted use, distribution, and reproduction in any medium, provided the original authors and source are credited. 